\title{
An X-ray view of Sagittarius $\mathrm{C}$
}

\author{
D. Chuard ${ }^{1,2}$, R. Terrier ${ }^{2}$, A. Goldwurm ${ }^{1,2}$, M. Clavel $^{3}$, \\ S. Soldi ${ }^{2}$, G. Ponti ${ }^{4}$, M. R. Morris ${ }^{5}$ and C. Jin ${ }^{4}$ \\ ${ }^{1}$ IRFU/Service d'astrophysique, CEA Saclay, 91191 Gif-sur-Yvette Cedex, France \\ ${ }^{2}$ APC, Univ. Paris Diderot, CNRS/IN2P3, CEA/Irfu, Obs. de Paris, USPC, France \\ ${ }^{3}$ Space Sciences Laboratory, University of California, Berkeley, CA 94720, USA \\ ${ }^{4}$ Max-Planck-Institut für extraterrestrische Physik, 85748, Garching, Germany \\ ${ }^{5}$ Dep. of Physics and Astronomy, University of California, Los Angeles, CA 90095, USA \\ email: dimitri.chuard@cea.fr
}

\begin{abstract}
Over the past 15 years, the molecular complex Sgr C has been repeatedly observed with both XMM-Newton and Chandra. These observations reveal new features indicating that the region might be more complex than previously thought. We find that its strong iron line emission at $6.4 \mathrm{keV}$ varies significantly over time, which supports the X-ray reflection scenario.
\end{abstract}

Keywords. Galaxy: center, ISM: reflection nebulae, X-rays: ISM

\section{The molecular complex Sagittarius $\mathrm{C}$}

The molecular complex Sagittarius C (Sgr C) is located approximately 0.5 degree away from the centre of the Milky Way. This is a key position to investigate the high-energy processes ongoing in the central molecular zone and their link with the past activity of the supermassive black hole Sgr A* (Ponti et al. 2013). Sgr C has therefore been repeatedly observed with various X-ray observatories. Suzaku notably revealed that it hosts a supernova remnant (SNR) candidate associated with a chimney-like outflow structure bright in the Sxv K $\alpha$ line (Tsuru et al. 2009). Observations performed with Chandra and XMM-Newton between 2000 and 2014 are now unveiling a more complex morphology for its thermal diffuse emission. In particular, using Herschel far-infrared data to trace the dense gas (Molinari et al. 2011), we find that the spatial correlation between molecular material and the edge of the SNR candidate G359.41-0.12 reported by Tsuru et al. (2009) is actually limited to the Galactic-northeastern portion of the SNR (Fig. 1, left). This indicates that its sharp, boomerang-like shape cannot be fully explained neither by absorption nor by dense gas preventing the plasma to expand further out. Besides, we discover a non-thermal comet-like X-ray feature within 30 arcsec of G359.40-0.08, another cometary X-ray filament already identified by Johnson et al. (2009). Their morphology and spectrum indicate that they are likely pulsar wind nebulae. Their proximity to each other and to the SNR candidate is puzzling and suggests that the origin of the Chimney is more complex than a simple outflow associated with the SNR. It also indicates that G359.41-0.12 itself may be more than a single SNR, as has already been suggested by Ponti et al. (2015).

\section{Variability of the iron $\mathrm{K} \alpha$ line}

The non-thermal diffuse emission from Sgr C consists of a Compton reflection continuum and a strong Fe K $\alpha$ line at $6.4 \mathrm{keV}$. Chandra and XMM-Newton observations show that its morphology is similar to previous descriptions based on Suzaku data (Nakajima et al. 2009, Ryu et al. 2013). They also reveal a short-term variability together with an apparent motion of the Fe K $\alpha$ emitting features towards the Galactic west (Fig. 1, right). 

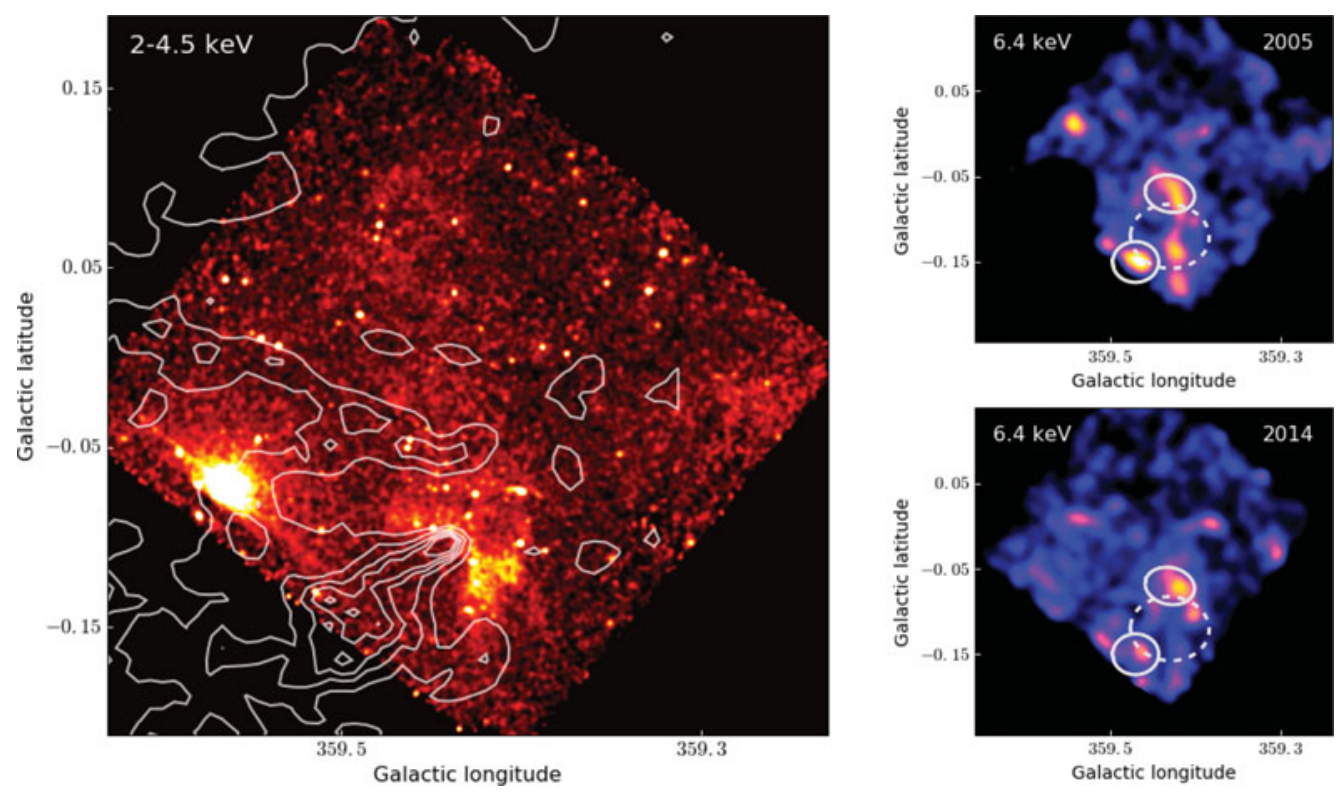

Figure 1. Chandra view of $\mathrm{Sgr} \mathrm{C}$ in the $2-4.5 \mathrm{keV}$ and $6.4 \mathrm{keV}$ bands. The bright clumps M359.43-0.07 and M359.47-0.15 are marked by the solid ellipses. The dotted ellipse indicates the region G359.41-0.12 used to estimate the local diffuse emission. Hi column density contours inferred from Herschel data are overlaid on the $2-4.5 \mathrm{keV}$ image.

This short-term variability is comparable to what has been observed in Sgr B2 and in the Sgr A complex (Terrier et al. 2010, Ponti et al. 2010, Clavel et al. 2013). In order to study it more quantitatively, we determine the flux in the Fe K $\alpha$ line by fitting spectra extracted in the main regions identified by Nakajima et al. (2009): two bright clumps spatially associated with molecular clouds (M359.43-0.07 and M359.47-0.15) and G359.41-0.12 that is used as a control region. After blank-sky spectra subtraction, spectral fits are carried out using a model composed of two thermal plasmas with $k T \sim$ $1 \mathrm{keV}$ and $k T \sim 7 \mathrm{keV}$, an absorbed power-law and an unabsorbed gaussian line at 6.4 $\mathrm{keV}$. The results reveal a statistically significant time variability in all three regions (at a $99 \%$ confidence level). This strongly suggests that the $6.4 \mathrm{keV}$ emission from Sgr C is due to X-ray reflection, probably due to past flares from $\operatorname{Sgr} A^{\star}$, rather than to cosmic-ray irradiation, even if we cannot rule out that low-energy cosmic rays might contribute to the much fainter local diffuse emission.

\section{References}

Clavel, M., Terrier, R., Goldwurm, A. et al. 2013, A\&A A, 558, A32

Johnson, S. P., Dong, H., \& Wang, Q. D. 2009, MNRAS, 399, 1429

Molinari, S. , Bally, J., Noriega-Crespo, A. et al. 2011, ApJ, 735, L33

Nakajima, H., Tsuru, T. G., Nobukawa, M. et al. 2009, PASJ, 61, S233

Ponti, G., Terrier, R., Goldwurm, A. et al. 2010, ApJ, 714, 732

Ponti, G, Morris, M. R., Terrier, R. et al. 2013, Astrophys. Space Sci. Proc., 34, 331

Ponti, G., Morris, M. R., Terrier, R. et al. 2015, MNRAS, 453, 172

Ryu, S. G., Nobukawa, M., Nakashima, S. et al. 2013, PASJ, 65, 33

Sunyaev, R., Markevitch, M., \& Pavlinsky, M. 1993, ApJ, 407, 606

Terrier, R., Ponti, G., Bélanger G. et al. 2010, ApJ, 719, 143

Tsuru, T. G., Nobukawa, M., Nakajima, H. et al. 2009, PASJ, 61, S219 